Canad. Math. Bull. Vol. 20 (4), 1977

\title{
LIE ALGEBRA AS A UNIFYING CONCEPT IN MODERN PHYSICS
}

\author{
BY \\ EDWIN IHRIG
}

\begin{abstract}
Lie algebras, in the form of algebras of observables, play an essential role in the formulation of classical and quantum mechanics. We discuss whether lie groups play a similar role in general relativity through the holonomy group. We also explore what interrelations these ideas provide between classical physics, relativity and quantum physics.
\end{abstract}

1. Introduction. Groups have played an important role in the development of physics. Since simple physical problems often enjoy symmetry which is independent of the mathematical models used to represent them, it is not surprising to find that symmetry groups are useful in very diverse theories. Thus in quantum mechanics the problem of the motion of non-interacting electrons around a nucleus can be solved by using the representation theory of $0(4)$ [24], the symmetry group. In general relativity the problem of finding the motion of non-interacting planets around a star can be solved with the help of the restrictions placed on the gravitational potentials by the symmetry group $0(3) \times \mathbb{R}[22]$. Of course the methods of using the symmetry groups in these two cases are quite different because the mathematical formalisms are so dissimilar. One of the appealing aspects of symmetry groups is that their applicability does not depend on the type of mathematical model used. However they have the limitation that they only are useful in simple nonrealistic problems. Also their most important limitation is that they serve only as a mathematical tool for solving equations of state, and they do not seem to play an essential role in the formulation of the physical theory.

In quantum mechanics there is another group that enters in a more fundamental way than do symmetry groups. This group occurs by way of its associated lie algebra. In both classical mechanics and quantum mechanics the observables can be seen to form a lie algebra (see the appendices for a brief outline of the relevant features of these theories). The equations of state governing the time evolution of observables can be formulated completely within these lie algebras. Thus lie algebras play a fundamental role in the foundation of these subjects.

Received by the editors August 10, 1977.

This paper is one of a series of survey papers written at the invitation of the Editors of the Canadian Mathematical Bulletin. 
Moreover the lie algebra of observables in quantum mechanics can be exploited to clarify and solve problems that would be otherwise intractable. In considering complicated physical systems many observables enter into the theory. For example in an atom, nucleus or crystal there are $3 n$ position and $3 n$ momentum observables where $n$ is the number of particles. However, only a certain few collective observables which give general measures of the state of the whole object in question are of interest. The problem in which all possible observables are included is intractable. However if the collective observables form a subalgebra $\delta$ of the algebra of observables and if the equations of state are well defined on this subalgebra i.e.

$$
[H, \delta] \subset \delta
$$

(see A2.5) then one may remove all the other extraneous variables from consideration. As the states in quantum mechanics are rays in a Hilbert space on which the lie algebra of observables acts, one then needs only to study the irreducible representations of $\delta$ which occur in the usual quantum mechanical representation to be able to construct a quantum theory of collective motion. Several models of collective motion have been well known (for example the rotational and liquid drop models [3], [4]), but it has not been known how to relate these models to quantum mechanics. Algebraic formulations of these models in the manner described above have been given by Rosensteel et. al. (see [18], [20] or [21] for details). This provides a theoretical justification for existing models, and it also enables the further development of existing models and the creation of more comprehensive models (see [18]). As an example of the further development that is possible, the quantum justification of the Bohr model gives, for the first time, an expression for the kinetic energy which is correct under all circumstances [19]. So quantum mechanics is endowed with an algebraic structure which plays an important role in the foundation of the theory as well as in the solution of practical problems.

In this paper we would like to discuss the possibility of there being an analogue to this algebra in general relativity. As general relativity can be considered a classical system with additional structure, the classical algebra of observables is still present. However its practical usefulness is lost in that there is no preferred finite dimensional subalgebra, such as the Heisenberg subalgebra in quantum mechanics (see A.2.3), due to its coordinate invariant formulation. Even the full algebra of classical observables is of questionable theoretical importance because the connection between this algebra and the fundamental physical quantities (the metric and curvature) seems very tenuous, if present at all.

However there is a natural group present in relativity-the holonomy group. We will consider its relationship with the metric, and discuss whether it can provide any further insight into the structure of relativity. We will also explore 
relationships between this group and the algebra of observables. Perhaps the results indicated may serve to suggest that the holonomy group has theoretical importance in relativity comparable to that of lie algebras of observables in quantum physics. In fact, it may turn out that this group will provide a means for forming a unified picture of classical, quantum and relativistic physics.

The appendices are provided to give a brief self-contained presentation of the aspects of modern physics we will be concerned with. More details may be found in [1] (classical mechanics), [6] (quantum mechanics) and [17] (relativity). A general knowledge of differential geometry ([9], [15]), and lie group theory ([2], [14], [16]) is assumed.

2. The holonomy group in general relativity. We would like to explore whether the holonomy group plays any special role in the foundations of relativity. A general knowledge of differential geometry ([9], [15]), and lie group theory ([2], [14], [16]) is assumed.

2.1. Definition. Let $(M, g)$ be a spacetime.

(a) If $\gamma$ is a path from $m_{0}$ to $m_{1}$ and $v \in T_{m_{0}}(M)$ let ${ }_{\gamma} T v \in T_{m_{1}}(M)$ be the result of parallel transport of $v$ along $\gamma$ using the connection $\nabla$ associated with $g$.

(b) The holonomy group $\phi_{m}$ is defined by $\phi_{m}=\left\{{ }_{\gamma} T / \gamma\right.$ is closed path from $m$ back to itself\}. Sometimes just $\phi$ will be used when the base point is not important.

The holonomy group is a lie group and forms a subgroup of the lorentz group $0(3,1)$ because

$$
g\left({ }_{\gamma} T v,{ }_{\gamma} T w\right)=g(v, w)
$$

This is due to the fact that $\nabla g=0$. The first question that should be resolved is whether the holonomy group embodies all the structure in a spacetime. In other words we must ask whether the holonomy group determines the metric. The answer is yes in a certain sense, as long as the generic situation in which the holonomy group's identity component is that of the full lorentz group is considered. If there are two metrics $g$ and $g^{\prime}$ such that ${ }_{\gamma} T={ }_{\gamma} T^{\prime}$ for any loop from $m$ to itself ( $m$ fixed) then $g=K g^{\prime}$ where $K$ is a constant (see [10] 3.5). As the constant $K$ has no effect on geodesics, $g$ and $g^{\prime}$ produce the same physical picture. An interesting offshoot of this idea is that under certain generic conditions the Riemann curvature tensor $R_{i j k}^{l}$ also determines $g_{i j}$ to within a constant conformal factor ([10]2.2). This arises from the fact that the set

$$
\left\{R(x, y) / x, y \in T_{m}(M)\right\}
$$

generates a sub lie algebra of the lie algebra of $\phi$ under the usual product 
$[A, B]=A B-B A$ where $A, B: T_{m}(M) \rightarrow T_{m}(M)$. Under generic conditions this subalgebra is the whole lie algebra of $\phi$, and since the lie algebra of a group determines that group's identity component (at least to within a covering), this theorem is quite natural. However this theorem is not strictly a corollary of the first theorem because the first result requires more than knowledge of what the holonomy group is but also knowledge of the correspondence between paths and holonomy elements. A striking aspect of this uniqueness theorem is that it is constructive. It permits one to find $g_{i j}$ from $R_{i j k}^{l}$ by solving a first order linear equation in one unknown function. This is a considerable simplification of this problem in that the curvature is a non-linear second order function of the 10 metric functions $g_{i j}$. Of course these results would be more interesting if they could be extended to Ric since Einstein's equations (see A 3.3) essentially specify Ric in terms of a given energy tensor T. A partial result (see [11]4.4) is available, however the basic question of whether Ric determines $g_{i j}$ and if so, how, remains unsolved. This question in itself is very fundamental in relativity and the light the holonomy group sheds on it is an indication of the group's potential significance to the field.

We have seen that just as the lie algebra of observables can be used to determine the physical states, so too the holonomy group can be used to determine the metric and thus the geodesics which are the physical states in relativity. A natural question that arises is whether the group structure is significant. exp gives a correspondence between every lie algebra element and every group element in the identity component. Thus we are asking whether the holonomy group may not be connected, and if so whether this has any physical significance. To answer the first question we produced a series of models for which the holonomy group is $\mathbb{Z}-$ a discrete group (see [12]). These models are locally flat $\left(\mathbb{R}_{i j k}^{l}=0\right.$ everywhere). In these models one discovers that light following certain paths will become redshifted. In fact this redshift can be associated with the holonomy group. If one then considers a generic subcollection of the collection of models for which this type of redshift effect is the only one present, one is able to find the distribution of these redshifts (see [13]). It has several unusual properties. One is that the distribution is discrete. This arises from the fact that these redshifts are associated with $\phi / \phi^{0}$ where $\phi^{0}$ is the identity component of $\phi$. Another is that the effect is strongest in one spatial direction and its antipodal direction. Thus this gives rise to an unusual new type of redshift effect. Certain physical objects, quasi-stellar objects, may have some of these characteristics ([15]). Whether they do or not, $\phi$ 's group structure provides a new tool in understanding the nature of redshift in relativity, and in the process gives a fuller understanding of the complete gamut of redshifts possible in this theory. It is also interesting to remark that this particular application has physical importance that reaches beyond understanding redshift phenomena. It provides us with an observational way of 
detecting that the universe has non-trivial topological structure. If such a discrete distribution of redshifts were observed then space could not be simply connected. This is so because there is a map from $\pi_{1}(M)$ to $\phi / \phi_{0}$ (a closed loop $\gamma$ goes to the class of ${ }_{\gamma} T$ ). Since this map is onto and $\phi / \phi_{0}$ is not 0 (in fact it can not even be torsion) $\pi_{1}(M)$ can not be zero. Since the relationship between the topological structure of the universe and locally observable phenomena is very subtle indeed, there are very few ways known of actually detecting what the universe's topology is. Detecting information about the fundamental group is even more difficult in so far as almost all physical quantities known are invariant under local isometries such as covering projections. In so far as this effect may shed light on the observability of topological structure, it plays a significant role in the foundations of relativity. Thus the whole group $\phi$, and not just its lie algebra, does have physical importance.

However, the most central question is whether the holonomy group has some relationship to the lie algebra of observables. There is in fact a connection which we are now starting to explore. The holonomy group (as well as the full lorentz group) at a point $m$ acts on $T_{m}(M)$. This action can be made into an action of a neighbourhood of $m$ in $M$ using the map $\exp ^{D}$ to identify vectors in $T_{m}(M)$ with points in $M$ ( $\exp ^{D}$ will denote the exp of differential geometry). Thus each element in the lie algebra of $\phi$ gives rise to a local vector field in $M$ which is the lie derivative of this action. A vector field $v$ on $M$ is a special kind of classical observable; that is, $v$ maps $P=T^{*}(M)$ into $\mathbb{R}$ simply by

$$
v(\theta)=\theta(v) \quad \theta \in P
$$

Thus each element $x$ of the lie algebra of the lorentz group at the point $m$ (denoted $l_{m}$ ) has a local classical observable $i(x)$ associated with it. We summarize this in the following definition.

2.2 Definition. Let $x \in l_{m}$, the lie algebra of the group

$$
L_{m}=\left\{A \mid A: T_{m}(M) \rightarrow T_{m}(M) \quad \text { and } \quad g(A v, A w)=g(v, w) v v, w\right\}
$$

then

is defined by

$$
i(x): P=T^{*}(M) \rightarrow \mathbb{R}
$$

$$
\left.i(x) \theta=\theta\left(D\left(\exp ^{D}\right)\left(\left.\sum_{i} \frac{d}{d t}(\exp t x) \xi\right|_{t=0}\right)_{i} \frac{\partial}{\partial \xi_{i}}\right)\right)
$$

where $\xi_{i}$ is a linear coordinate system for $T_{m}(M)$.

Notice that $i$ is a one to one lie algebra homomorphism into the algebra of vector fields. Let us look at the case $M=\mathbb{R}^{4}$ with the metric $d s^{2}=$ $d x^{2}+d y^{2}+d z^{2}-d t^{2}$ (Minkowski space). Then $\bigcup_{n \in M} i\left(l_{m}\right)$ is a finite dimensional lie algebra. Its group is the Poincare group, the group of all transformations from $\mathbb{R}^{4}$ to itself that preserves $x^{2}+y^{2}+z^{2}-t^{2}$. It is the lorentz 
group plus transtations. The generators of the lorentz group give rise to the angular momentum observables $x_{i} p_{j}-x_{j} p_{i} \quad i, j \neq 0$ and $x_{0} p_{i}+x_{i} p_{0}$. The generators of the transtations give rise to the momentum observables $p_{i}$. Thus we see that the metric structure on Minkowski space allows us to pick out the appropriate momentum and angular momentum observables. With this information the position observables could be extracted using the fact that they are 'canonically conjugate' to the momentum i.e.

$$
\left\{x_{i}, p_{j}\right\}=\delta_{i j}
$$

Thus the metric has provided us with sufficient information to find the appropriate kinematical observables. This gives us a precise way of using general relativity to find the finite dimensional subalgebra of observables which is necessary to formulate a quantum mechanical system.

In the case of a general spacetime the map $i$ manages to define angular momentum about the point $m$. This has been done previously only with the help of symmetries [8]. Although angular momentum usually arises as a symmetry in free space, it is essentially a kinematical quantity which should not rely on symmetry for its definition but only for its conservation. Thus this definition seems to be more satisfactory from a physical point of view in that it only requires the kinematical structure of relativity for its use. As isometrics commute with $\exp ^{D}$, this definition reduces to the usual one when symmetry is present.

Two critical questions arise immediately. The first is what conditions are necessary to enable $\bigcup_{m \in M} i\left(l_{m}\right)$ to be a finite dimensional lie algebra. Can one extract linear momentum in this case? If these conditions are reasonable then one will have a precise method of taking the coordinate dependence out of quantum mechanics. Classical mechanics together with the kinematical information provided by a relativistic structure will enable one to pick the appropriate subalgebra of observables to be dealt with in quantum mechanics (see appendix 2).

The second question is whether giving the system of $\bigcup_{m \in M} i\left(l_{m}\right)$ is equivalent to giving a spacetime structure. In other words does $\bigcup_{m \in M} i\left(l_{m}\right)$ uniquely determine the metric? It is obvious that $\bigcup_{m \in M} i\left(l_{m}\right)$ determines $g$ to within a (not necessarily constant) conformal factor. This follows because $l_{m}$ determines $g$ to within a constant factor at $m$ due to Schur's lemma, and $i\left(l_{m}\right)$ determines $l_{m}$ by means of looking at $\left(D \rho_{t_{0}}\right) m$ where $\rho_{t}$ is the integral of $i(x)$ and $m$ is the fixed point of $\rho_{t_{0}}$. The only question lies in the conformal factor, and it seems likely that the only source of ambiguity will be a constant conformal factor which is insignificant from the physical point of view. If this is so then one may take a different viewpoint of relativity. Relativity could then be seen as a prescription which gives the angular momentum observables about each point in the classical configuration space. Relativity theory would then be a rigorous 
way of prescribing the relevant kinematical observables in classical systems from the physical content of the universe.

If both of these questions could be answered to the affirmative; that is, if relativity is characterized by a specification of angular momentum observables and if these observables are all that is needed to extract all the relevant kinematical observables, then the role of relativity in physics might seen quite different. Relativity with its cosmological applications may not seem as being quite so remote from microscopic quantum physics. Rather it may play the role of a bridge between classical and quantum mechanics in that it finds the finite dimensional subalgebra of classical observables that enables one to proceed with the formulation of quantum mechanics. If such a unifying concept can be justified, insight into the strengths and weaknesses of modern physics may be easier to decipher. Thus lie algebras of observables with the help of the holonomy group may indeed enjoy a very important role in the structure of modern physics.

Appendix 1, Classical mechanics. The following is a brief description of classical mechancis (see [1] for more details). A particle or system of particles is assumed to have a position describable by a point in a manifold $\boldsymbol{M}$ called the configuration space. For a single particle allowed to travel freely in space $\boldsymbol{M}$ would be $\mathbb{R}^{3}$. For a particle attached to the end of a rigid pendulum the configuration space would be $S^{2}$, the two dimensional sphere. Configuration space for $n$ freely moving particles would be $\mathbb{R}^{3 n}$. As both position and momentum are needed to characterize the motion of particles, one is actually interested in $P=T^{*}(M)$ which is called phase space or momentum space. There is a natural two form $\omega$ on $P$ defined in the following way. If $x_{i}$ are local coordinate system in $M$, any one form $\theta$ is expressable as

$$
\theta=\sum_{i=1}^{n} p_{i} d x_{i}
$$

One can verify that

$$
\begin{gathered}
\phi: T^{*}(M) \rightarrow \mathbb{R}^{2 n} \\
\phi(\theta)=\left(x_{1}, \ldots, x_{n}, p_{1}, \ldots, p_{n}\right)
\end{gathered}
$$

forms a local coordinate system for $T^{*}(M)$. Thus

$$
=\sum_{i=1}^{n} d x_{i} \wedge d p_{i}
$$

is a closed, non-singular two form defined on an open set in $P=T^{*}(M)$. It can be shown that $\omega$ does not depend on the coordinate system used so it may be extended to a global form on $P$. 
Now that we have $P$ and $\omega$, we have all the ingredients necessary to define classical mechanics.

A1.1 Definition Let $P$ be a manifold with a closed non-singular two form $\omega$.

(1) An observable on $P$ is a function $f: P \rightarrow \mathbb{R}$

(2) $\#: T^{*}(M) \rightarrow T(M)$ is given by $\#=\mathfrak{b}^{-1}$ where $b: T(M) \rightarrow T^{*}(M)$ $(\mathrm{b}(v)) x=\omega(v, x)$

(3) If $f$ and $g$ are observables then the poisson bracket of $f$ and $g$ is a new observable defined by

$$
\{f, g\}=\omega(\#(d f), \#(d g))
$$

Some examples of observables are $\sum_{i} p_{i}^{2}$-the kinetic energy, $\sum x_{i}^{2}$-the potential energy for the harmonic oscillator, or $x_{i} p_{j}-x_{j} p_{i}$-the angular momentum. Notice that the set of observables together with the poisson bracket form a lie algebra (which is infinite dimensional) because $\omega$ is closed. We now define the second ingredient needed in a physical theory-the collection of states. A state is that element in the mathematical theory which characterizes all the relevant physical properties of the objects under study. In classical mechanics an object is completely specified by its position and momentum at all time. Thus we make the following definition:

A1.2. Definition. (a) A state is a smooth path $\gamma: \mathbb{R} \rightarrow P$

(b) If $f$ is an observable and $\gamma$ is a state then fo $\gamma: \mathbb{R} \rightarrow \mathbb{R}$ is the function which gives for each time $t$ the result of making the observation $f$ on the state $\gamma$ at time $t$.

We now have observables, states and the method of making observations. All we need are the equations of state which determine what states represent actual physical processes.

A1.3. Definition. Let $h$ be the observable (called the hamiltonian) which takes a point in $\boldsymbol{P}$ to its energy. Then the equations of state are

$$
\stackrel{8}{f}=\{f, h\}
$$

for all observables $f$. Here $f$ is $d(f o \gamma) / d t$; that is, $f$ is $D_{\gamma} f$, the directional derivative of $f$ along $\gamma$.

One can easily verify that this equation reduces to saying that $\#(d h)$ is the tangent field to $\gamma$. Thus $\gamma$ is uniquely specified given initial conditions (i.e. $\gamma(0)$-the initial position and momentum of the system). The advantage of leaving the equation in the form stated is that in this form the time evolution of a system is manifestly determined by the structure of the lie algebra of observables. 
Appendix 2, Quantum mechanics. The principal difference between classical mechanics and quantum mechanics lies in the states and not in the observables. The set of observables is assumed to be a lie algebra. This assumption can be justified in terms of basic properties of observables with the help of a few theorems (see [7]). The Dirac quantization procedure requires that this lie algebra actually is the same as the lie algebra of classical observables (see [6]). However this is not possible because of the nature of the state space which we will describe below (see [23]). In practice this difficulty is avoided by restricting attention to a subalgebra of classical observables that is small enough so that problems with quantum mechanical state space do not arise. We will give a concrete example of this later.

A2.1. Definition. In quantum mechanics the lie algebra of observables is a subalgebra of the classical lie algebra of observables.

We now need to define a state.

A2.2. Definition. A state is a path in the ray space of a Hilbert space $\aleph$. Moreover the elements of the lie algebra of observables are assumed to act on $\aleph$ as skew adjoint operators.

It can be shown there is no space $\aleph$ as above which carries a representation of the whole lie algebra of observables [23]. We give an example which is called the Heisenberg representation and which is the standard form of quantum mechanics used in physics.

A2.3. ExAmple. We consider an $n$ particle system. Thus the appropriate classical phase space is

$$
T^{*}\left(\mathbb{R}^{3 n}\right)=\mathbb{R}^{6 n}
$$

This has a natural chart described by $x_{i \alpha}$ and $p_{i \alpha}$ where $1 \leq i \leq n$ and $1 \leq \alpha \leq 3$. The set

$$
\left\{x_{i \alpha}, p_{i \alpha}, 1\right\}
$$

consisting of these linear projections and the constant function 1 is a subalgebra of the classical lie algebra of observables for this system. It is called the Heisenberg lie algebra. This is the algebra of observables for this example. We now describe the set of quantum mechanical states. Let $\aleph=\mathscr{L}^{2}\left(\mathbb{R}^{3 n}, \mathbb{C}\right)$ be the set of square integrable complex functions of $\mathbb{B}^{3 n}$. This is a Hilbert space with inner product

$$
(\psi, \phi)=\int_{R^{3 n}} \psi \bar{\phi}, \quad \psi, \phi \in H
$$


Now we define the action of the observables on this state space by

$$
\begin{aligned}
& \left(x_{i \alpha}(\psi)\right)\left(x_{i \alpha}\right)=i x_{i \alpha} \psi\left(x_{i \alpha}\right) \\
& \left(p_{i \alpha}(\psi)\right)\left(x_{i \alpha}\right)=\left(\frac{\partial}{\partial x_{i \alpha}} \psi\right)\left(x_{i \alpha}\right) \\
& (1 \cdot(\psi))\left(x_{i \alpha}\right)=i \psi\left(x_{i \alpha}\right)
\end{aligned}
$$

this gives a skew adjoint lie algebra action on $\aleph$.

Our last task is to describe how to make observations and what the equations of state are. Quantum mechanics is not a deterministic theory in so far as it does not predict exactly what the outcome of an observation of a state will be. It simply says the observation of a fixed state with a fixed observable will have a distribution of results whose mean is predictable. This mean is called the expected value of the observable.

A2.4. Definition. The expected value of the observation of a state $\bar{\psi}$ by an observable $A$ is given by

$$
i(\psi, A \psi) \mid(\psi, \psi)
$$

where (,) is the inner product and $\psi$ is any vector in the ray $\bar{\psi}$.

The fact that $A$ acts as a skew adjoint operator simply insures that the expected value of an observation will be a real number. All known laboratory methods of observation give real numbers as results; this is the reason the observables are assumed to act as skew adjoint operators. ${ }^{*}$

We now will give the equations of state in quantum mechanics. They are completely analogous with the classical equation of state.

A2.5. Definition. Let $H$ represent the energy observable of the system. If $A$ is any other observable and [,] denotes the lie product in the set of observables then

$$
\stackrel{\AA}{A}[A, H]
$$

where $\AA$ is defined by

$$
i \frac{\left(\psi_{t}, \AA \psi_{t}\right)}{\left(\psi_{t}, \psi_{t}\right)}=\frac{d}{d t}\left(i \frac{\left(\psi_{t}, A \psi_{t}\right)}{\left(\psi_{t}, \psi_{t}\right)}\right)
$$

Here $\bar{\psi}$. is any state and $\psi$. is any path from $R$ into $\aleph$ such that $\psi_{t}$ lies in the ray $\bar{\psi}$ for all $t$.

* One could require the action to be self adjoint and thus eliminate $i$ 's from the formulae. However such an action is not possible since self adjoint operators do not form a real lie algebra as do the skew adjoint operators. 
In the last part of this definition, the observation of $\AA$ on any state $\bar{\psi}$ is defined to be the time derivative of the observation of $A$ made on $\bar{\psi}$, just as in classical mechanics.

Appendix 3, General relativity. From the point of view of physical applications general relativity and quantum mechanics stand as the two pillars of modern physics. Relativity describes cosmological physics while quantum mechanics describes microscopic physics. Classical mechanics sits between these two pillars and enjoys a range of applicablility which is in the intersection of the ranges of these two.

However from the point of view of the structure of physical theories, relativity enjoys a less special role. In fact it may be considered as a restricted example of classical mechanics. It is concerned only with the problem of kinematics in a classical system, i.e. the motion of particles when no forces are present. It is perhaps fortuituous that gravitation turns out to be a 'fictional force' in so far as it seems to be completely describable in the kinematical setting of general relativity. As gravitational considerations predominate in cosmology, this gives relativity its important role in physical applications.

Why is kinematics a problem in classical mechanics? Of course everything needed to deal with a classical problem is available once one knows $h$ (see A1.3). For a system with no forces $h$ is just $\sum p_{i}^{2}$-the kinetic energy. However this function involves the coordinate projections. If another coordinate system is chosen then a different $h$ will occur. This will give a different equation of state. In fact one can easily use A1.3 to find

$$
\check{x}_{i}=c_{i} \quad c_{i} \text { constant. }
$$

Thus particles with no forces on them travel in straight lines in the coordinate system which is used to define $h$. How does one pick out this special coordinate system?

General relativity takes the following approach. It assumes that free particles travel on straight paths. in order to specify 'straight' the added structure of a pseudo*-metric is needed.

A3.1. Definition. A spacetime $(M, g)$ consists of a 4-dimensional manifold $M$ (the classical configuration space with time added) and a pseudo-metric $g$ with signature +++- (which is called a lorentz metric or a gravitational potential).

A3.2. Definition. (a) The states and observables for a spacetime are the same as the states and observables for the classical configuration space $M$.

(b) The equation of state for $\gamma$ are the geodesic equations for $\gamma$ in $g$; that is,

$$
\nabla_{\gamma_{*}} \gamma_{*}=0
$$


where $\nabla$ is the unique connection with

$$
\nabla g=0, \operatorname{Tor} \nabla=0 .
$$

Note that these equations of state may be related to those of classical mechanics by observing that the pseudo-metric $g$ defines a vector field in $T^{*} M$ whose integral curves project to the geodesics of $g$ in $M$. This vector field is the hamiltonian vector field $H$ which is related to $h$ by $\# d h=H, h=g$.

Of course, the main question remains: How does one determine $g$ ? $g$ must be determined by some physical aspect of the universe. The distribution of energy throughout the universe is perhaps the most obvious candidate for this task, if not the only one. This energy is characterized by a symmetric rank 2 tensor $T$. We now need only relate this to $g$. We let $R(\cdot, \cdot) \cdot$ (or just $R$ when no ambiguity arises) denote the Riemann curvature of $\nabla$ in A3.2. Ric is the nonzero trace of the Riemann curvature. $R$, called the scalar curvature, is the trace of Ric obtained with the help of the isomorphism induced by $g$ from $T(M)$ to $T^{*}(M)$.

A3.3. Definition. Let $(M, g)$ be a spacetime. The equations that determine $g$ in terms of the energy tensor $T$ are

$$
\operatorname{Ric}+\frac{1}{2} R g+\Lambda g=T
$$

where $\Lambda$ is a constant and $R$ is the scalar curvature.

These equations, known as Einstein's equations, enable one to determine $g$. This gives the kinematics of a classical system once the matter distribution of the universe is known.

\section{REFERENCES}

1. R. Abraham, (1967), Foundations of Mechanics, Benjamin, New York.

2. J. F. Adams, (1969), Lectures on Lie Groups, Benjamin, New York.

3. A.. Bohr, (1952), The Coupling of Nuclear Surface Oscillations to the Motion of Individual Nucleons, K. Danske Vidensk. Selsk, Mat-Fys. Medd. 26 No. 14.

4. Å. Bohr and B. Mottelson, (1953), Collective and Individual Particle Aspects of Nuclear Structure, K. Danske Vidensk. Selsk, Mat-Fys. Medd. 27 No. 16.

5. Burbidge G., (1968), The Distribution of Redshifts in Quasi-Stellar Objects, N-systems, and Some Radio and Compact Galaxies Ap J. (letters) 154 L41.

6. P. A. M. Dirac, (1958), The Principles of Quantum Mechanics, Oxford University Press, Oxford.

7. G. Emch, (1972), Algebraic Methods in Statistical Mechanics and Quantum Field Theory, Interscience New York.

8. S. W. Hawking and G. F. R. Ellis, (1973), The Large Scale Structure of Spacetime, Cambridge University Press, Cambridge.

9. N. Hicks, (1971), Notes on Differential Geometry, Van Nostrand, London.

\footnotetext{
*Considerations of light propagation indicate that time must be included in the configuration space. The special nature of the time direction is reflected in the fact the metric has signature +++- . Since the metric is not positive definite we use the word 'pseudo' to call attention to this.
} 
10. E. Ihrig, (1975), The Uniqueness of $g_{i j}$ in Terms of $R_{i j k}^{l}$, Int. J. Theor. Phys. 14, p. 23.

11. E. Ihrig, (1976), The Holonomy Group in General Relativity and the Determination of $g_{i j}$ from $T_{j}^{1}$ G.R.G. 7, p.313.

12. E. Ihrig, A Local Relativistic Redshift Effect, to appear G.R.G.

13. E. Ihrig, Redshift Formulae for a Local Relativistic Redshift Effect, to appear.

14. N. Jacobson, (1962), Lie Algebras, Interscience, New York.

15. S. Kobayashi and K. Nomizu, (1963), Foundations of Differential Geometry, Vol. I, interscience, New York.

16. W. Miller, (1972), Symmetry Groups and Their Applications, Academic Press, New York.

17. C. W. Misner, K. S. Thorne, and J. A. Wheeler, (1970), Gravitation, Freeman and Co. San Francisco.

18. G. Rosensteel, (1975), On the Algebraic Formulation of Collective Models, Ph.D. Thesis, University of Toronto, Toronto, Ontario.

19. G. Rosensteel and E. Ihrig, Kinetic Energy in the Bohr-Mottelson Collective Model, to appear Phy. Rev.

20. G. Rosensteel and D. J. Rowe, (1976), The Algebraic CM(3) Model, Annals of Physics 96, p. 1.

21. G. Rosensteel and D. J. Rowe, (1976), The Sp. $(3, R)$ Model of Nuclear Collective Motion. Invited Talk, 5th International Colloquium on Group Theoretical Methods in Physics, Montreal.

22. K. Schwarzschild, (1916), Uber das Gravitationsfeld eines Massenpunktes nach der Einsteinschen Theorie, Sitzber. Deut. Akad. Wiss. Berlin, K1. Math-Physics, Tech., p. 189.

23. L. van Hove, (1951), Mem Acad. Roy. Belge., 26.

24. H. Weyl, (1930), Group Theory and Quantum Mechanics, Dover (reprint 1950), New York.

Department of APPlied Mathematics

MCMASTER UNIVERSITY

Hamilton, ONT., Canada L8S 4K1 